\title{
L’enseignement du droit aux « non-initiés " : le cas des sciences de l'information
}

\author{
Teaching Law to the "Uninitiated": The Case of Information \\ Science
}

\section{La enseñanza del Derecho a los «profanos»: las Ciencias de la Información}

\section{Marie Demoulin}

Volume 61, numéro 2-3, avril-septembre 2015

La formation en sciences de l'information

URI : https://id.erudit.org/iderudit/1032811ar

DOI : https://doi.org/10.7202/1032811ar

Aller au sommaire du numéro

Éditeur(s)

Association pour l'avancement des sciences et des techniques de la documentation (ASTED)

ISSN

0315-2340 (imprimé)

2291-8949 (numérique)

Découvrir la revue

Citer cet article

Demoulin, M. (2015). L'enseignement du droit aux « non-initiés » : le cas des sciences de l'information. Documentation et bibliothèques, 61(2-3), 68-75. https://doi.org/10.7202/1032811ar
Résumé de l'article

Cet article se présente comme un partage d'expérience, afin de nourrir la réflexion sur l'enseignement du droit à des non-juristes, en particulier dans les programmes de formation en sciences de l'information (SI). La première partie livre un aperçu de la place du droit dans ces programmes et des différentes manières d'intégrer le droit dans le parcours des étudiants en SI. La seconde partie se focalise sur la pédagogie du droit au sein de l'École de bibliothéconomie et des sciences de l'information (EBSI) de l'Université de Montréal, à travers les cours « Aspects juridiques des archives et de l'information » et "Sources d'information juridique ».
Tous droits réservés (c) Association pour l'avancement des sciences et des techniques de la documentation (ASTED), 2015
Ce document est protégé par la loi sur le droit d'auteur. L'utilisation des services d’Érudit (y compris la reproduction) est assujettie à sa politique d'utilisation que vous pouvez consulter en ligne. 


\title{
L’enseignement du droit aux «non-initiés » : le cas des sciences de l'information
}

\author{
Marie Demoulin, Ph. D. \\ Professeure adjointe \\ EBSI, Université de Montréal \\ marie.demoulin@umontreal.ca
}

\section{RÉSUMÉ | ABSTRACT | RESUMEN}

Cet article se présente comme un partage d'expérience, afin de nourrir la réflexion sur l'enseignement du droit à des non-juristes, en particulier dans les programmes de formation en sciences de l'information (SI). La première partie livre un aperçu de la place $d u$ droit dans ces programmes et des différentes manières d'intégrer le droit dans le parcours des étudiants en SI. La seconde partie se focalise sur la pédagogie du droit au sein de l'École de bibliothéconomie et des sciences de l'information (EBSI) de l'Université de Montréal, à travers les cours "Aspects juridiques des archives et de l'information » et "Sources d'information juridique ».

\section{Teaching Law to the "Uninitiated": The Case of Information Science}

The intent of this article is to nurture a discussion about the teaching of law to non-jurists, especially those registered in information science programmes. The first part of this article presents the place of legal education in the programmes and the different ways the law can be integrated into the students' curriculum. The second part focuses on the learning of law at the École de bibliothéconomie et des sciences de l'information (EBSI) of the Universite de Montréal, namely in the courses "Aspects juridiques des archives et de l'information" and "Sources d'information juridique".

\section{La enseñanza del Derecho a los "profanos»:} las Ciencias de la Información

Este artículo representa una oportunidad de compartir experiencias, con el propósito de fomentar la reflexión acerca de la enseñanza del Derecho a los "profanos» 0 «no juristas», especialmente a través de programas de formación en Ciencias de la Información (CI). La primera parte del libro presenta una perspectiva de la función del derecho en estos programas $y$ de las distintas maneras de integrarlo en la carrera de los estudiantes de CI. La segunda parte se centra en la pedagogía del derecho en la Escuela de Bibliotecología y Ciencias de la Información (EBSI) de la Universidad de Montreal, mediante los cursos "Aspectos jurídicos de los archivos y de la información" $y$ «Fuentes de información jurídica».

\section{Introduction}

A ux yeux des "profanes ", le droit présente souvent un caractère mystérieux et opaque. Cette perception est notamment causée par l'usage d'un jargon technique et spécialisé, qui semble réservé aux initiés et qui rend difficilement accessibles la législation, les décisions de jurisprudence et la littérature juridique. Pourtant, en tant que mode de régulation de la vie en société, le droit est partout et touche les personnes dans tous les aspects de leur vie, et notamment leur vie professionnelle. Quel que soit le secteur d'activité, les problèmes juridiques ne cessent de surgir sur le terrain et doivent souvent être gérés sans la présence d'un avocat, faute de temps ou de moyens. Partant de ce constat, un nombre croissant de programmes d'études supérieures intègrent dans la formation de leurs étudiants des aspects de droit destinés à les initier aux principales questions juridiques auxquelles ils pourraient être confrontés dans le cadre de leur activité. Cette tendance récente s'observe également dans les programmes de formation en sciences de l'information (SI), en raison d'une demande de plus en plus insistante des milieux professionnels pour une plus grande sensibilisation au droit.

La présente contribution vise à nourrir la réflexion sur l'enseignement du droit à des non-juristes, en particulier dans les programmes de formation en SI. La première partie esquisse les contours de l'enseignement du droit dans divers programmes en SI au Québec, au Canada ou ailleurs, afin d'illustrer différentes manières d'intégrer le droit dans le parcours des étudiants ${ }^{1}$. La seconde partie se focalise sur la pédagogie du droit au sein de l'École de bibliothéconomie et des sciences de l'information (EBSI) de l'Université de Montréal, à travers les cours "Aspects juridiques des archives et de l'information » et «Sources d'information juridique ». Cette partie se présente comme un partage de ma démarche pédagogique comme professeure de droit au sein d'une école de SI.

1. L'auteure tient à remercier chaleureusement Ivan Barreau pour l'aide précieuse apportée dans l'exploration de divers programmes francophones et anglophones en sciences de l'information, afin d'y déceler les éléments de droit qui y sont enseignés. 


\section{La place du droit dans les programmes de formation en sciences de l'information}

L’enseignement du droit se répand dans les programmes de formation en SI. En soi, il s'agit là d'une association logique, dans la mesure où information et droit convergent à plus d'un titre (Gathegi \& Burke 2008, 1). D'une part, le droit repose fondamentalement sur des ressources informationnelles, qu'il faut pouvoir trouver, analyser et gérer. D'autre part, le champ des SI est de plus en plus souvent confronté à des questions juridiques et éthiques (Fernández-Molina 2004, 111), telles que le droit d'auteur, la vie privée et la protection des renseignements personnels, l'accès à l'information, le droit à l'oubli, la censure, le contrôle et la surveillance de l'information, les technologies de l'information, les obligations légales de conservation de documents, sans oublier la législation propre au secteur lui-même : législation sur les archives et les bibliothèques, sur les livres et le secteur de l'édition, sur les médias et le secteur audiovisuel, la déontologie... On relève au passage que l'avènement de la société de l'information a exacerbé cette convergence en développant les bases de données juridiques et le droit de l'information.

Selon une enquête menée en 2008 auprès de 63 écoles nord-américaines de SI, $89 \%$ des responsables de programmes considéraient l'étude des questions juridiques comme primordiale (" critical \& highly critical ») pour l'avenir des SI (Gathegi \& Burke 2008, 9). Mais si les responsables de programmes en SI semblent manifester un intérêt croissant pour le droit, celui-ci n'est pas enseigné partout de la même manière ni avec la même intensité. Sans prétendre fournir ici une étude exhaustive ni quantitative des programmes offerts en la matière, il semble intéressant d'examiner la diversité des approches.

Notre analyse a porté sur des programmes universitaires en SI et en archivistique offerts en français et en anglais aux $1^{\mathrm{er}}$ et $2^{\mathrm{e}}$ cycles au Québec et dans certaines institutions du Canada, des États-Unis, du RoyaumeUni, de Belgique, de France et de Suisse, pendant l'année universitaire 2014-2015. Faute de moyens, l'étude s'est limitée aux programmes accessibles en ligne et aux descriptions de cours suffisamment détaillées. Elle a également pu s'appuyer sur les statistiques fournies par l'étude de Gathegi et Burke, menée en 2008, qui fournit des éléments intéressants (bien que non actualisés) sur les tendances en Amérique du Nord, et surtout aux États-Unis, dans la mesure où sur les 63 répondants, 56 étaient des écoles américaines, contre seulement sept écoles canadiennes.

On relève d'emblée qu'un cours spécifiquement dédié au droit n'est pas offert dans tous les programmes. Cela ne signifie pas que les aspects juridiques ne sont pas abordés au cours de la formation. En effet, de nombreux cours non juridiques traitent des éléments de droit et d'éthique, tant pour évoquer la législation applicable à la profession que pour examiner les grandes questions juridiques à laquelle celle-ci est confrontée. On songe à des cours d'introduction aux SI ou à l'archivistique, de gestion de l'information numérique, de politique ou de gouvernance informationnelle, d'éthique et de rapport du professionnel à la société... Il est donc assez courant que des éléments juridiques soient évoqués au sein des cours de SI, sans pour autant qu'un cours spécifiquement dédié au droit soit inscrit au programme. L'inconvénient de ces évocations dispersées est qu'il est difficile d'assurer la coordination des connaissances en vue de former un corpus complet et cohérent sur les aspects juridiques de l'information. L'intégration au programme d'un ou de plusieurs cours de droit permet de remédier à ce problème.

L'offre de cours est très variable sur le plan du contenu. Il peut s'agir d'un cours de droit de l'information et des archives ou de plusieurs cours spécialisés (droit d'auteur et propriété intellectuelle, vie privée et sécurité, droit de l'information du secteur public, droit des archives, sources juridiques et méthodes de recherche en droit, informatique juridique, bibliothèques spécialisées en droit, etc.). Gathegi et Burke (2008) résument bien la situation; ils identifient trois catégories de cours juridiques : le droit de l'information, la gestion de l'information juridique ainsi que l'éthique et la liberté intellectuelle. Cette dernière catégorie est liée au droit de manière plus indirecte, en abordant des questions éthiques (liberté intellectuelle, censure, etc.) sans nécessairement en faire l'objet central du cours (Gathegi \& Burke 2008, 4).

D'autres programmes offrent aux étudiants la possibilité de suivre des cours de droit plus généralistes et non spécifiquement dédiés au droit de l'information. Parmi ceux-ci, on retrouve des cours sur les fondements du droit, d'introduction au droit privé et au droit public, de droit constitutionnel, de droit administratif, de droit fiscal et commercial, de droit de propriété intellectuelle, de droit des médias, de l'information et de la communication et de droit de la culture. Chacune de ces matières est susceptible d'intéresser un professionnel de l'information, selon le secteur d'activité auquel il se destine.

Malgré un intérêt croissant pour le droit dans les milieux de l'information, on ne rencontre guère de programmes qui imposent un cours obligatoire dans cette matière. Ceux qui ne sont pas intéressés par le droit peuvent donc être diplômés sans nécessairement avoir suivi un cours de droit. Ceux qui, au contraire, ont un intérêt pour le droit doivent s'en tenir à un ou deux cours de droit choisis parmi les options ou filières disponibles, en raison de la configuration du programme et du nombre de crédits requis pour l'obtention du diplôme. On note cependant que des écoles se sont associées avec des facultés de droit pour proposer un diplôme conjoint en droit et en SI; cette tendance semble à la hausse aux 
États-Unis, mais elle reste rare au Canada et elle est inexistante au Québec ${ }^{2}$.

Selon les configurations, on trouve dans les programmes des cours de droit " maison » et des cours de droit accessibles dans d'autres facultés, le plus souvent la faculté de droit voisine, auxquels les étudiants en SI peuvent s'inscrire dans le cadre d'accords interfacultaires. Cependant, les cours offerts en faculté de droit sont destinés essentiellement à des étudiants en droit, et exigent parfois des prérequis en droit. On peut dès lors s'attendre à ce qu'ils ne soient pas adaptés à un public profane et, a fortiori, ne répondent pas aux besoins particuliers des SI. Parfois, les cours de droit sont destinés globalement à un public de non-juristes issus de diverses disciplines, englobant sciences économiques, politiques et sociales, les SI et la communication. Le propos est alors plus accessible, mais s'en tient nécessairement à une présentation généraliste de la matière, sans entrer dans les questions qui intéressent spécifiquement les professionnels de l'information et qui touchent à leur pratique courante.

Quelques programmes offrent un cours de droit sur mesure, conçu par un professeur du département et spécialement dédié aux étudiants en $\mathrm{SI}^{3}$. Cette option semble souhaitable pour pouvoir assurer une pédagogie adaptée. Il est clair que le droit ne s'enseigne pas de la même manière aux juristes (futurs praticiens du droit) et aux non-juristes (destinataires du droit). Il s'agit à la fois d'adapter les objectifs pédagogiques et le discours lui-même, en proposant un contenu cohérent qui corresponde aux besoins réels du métier. Cela étant, dans le cas particulier où un diplôme conjoint en droit et en SI est offert, l'étudiant se trouve plongé dans un programme véritablement juridique qui lui fournit la méthodologie et le contexte qui lui permettent d'assimiler facilement la matière, même si elle s'adresse principalement à des futurs avocats.

En résumé, d'après les programmes examinés, les questions juridiques relatives aux SI peuvent être dispersées dans des cours généraux ou concentrées dans un ou plusieurs cours de droit dont le contenu est variable (généraliste, droit de l'information, ou plus spécialisé encore). Dans l'offre de cours, on trouve des cours obligatoires, à options ou des programmes conjoints en droit et en SI, des cours de droit " maison » et des cours offerts dans d'autres facultés (souvent des facultés de

2. Selon Gathegi et Burke, parmi les 63 écoles nord-américaines ( 56 américaines et 7 canadiennes) interrogées en $2008,39,0 \%$ offraient déjà un programme conjoint en droit et en sciences de l'information, et 31,8 \% envisageaient de développer un tel programme $(2008,9)$. À supposer que ces projets se soient effectivement réalisés, cela signifierait qu'environ $70,0 \%$ de ces écoles pourraient potentiellement offrir de tels programmes aujourd'hui. Il serait bien sûr nécessaire de mener une nouvelle étude aujourd'hui afin de vérifier une hypothèse aussi optimiste. Au Canada, l'Université Dalhousie (Nouvelle-Écosse) est la première à avoir mis en place un diplôme conjoint en droit et en sciences de l'information, suivie par l'Université de Toronto.

3. Selon l'étude de Gathegi et Burke, 39,0\% des 63 écoles en sciences de l'information nord-américaines interrogées en 2008 avaient un diplômé en droit au sein de leur corps enseignant $(2008,9)$. droit). Ces cours s'adressent tantôt à des futurs praticiens du droit, tantôt à un public non-initié, voire spécifiquement aux futurs professionnels de l'information.

\section{L'enseignement du droit à l'EBSI : retour d'expérience}

Les programmes du certificat en archivistique, du certificat en gestion de l'information numérique et de la maîtrise en sciences de l'information (MSI) de l'EBSI ont conçu une offre de cours qui intègre davantage de notions juridiques dans la formation. Si l'on reprend les catégories de Gathegi et Burke (2008), on constate que l'EBSI offre désormais un cours de droit de l'information (ARV3053 - Aspects juridiques des archives et de l'information, cours à option offert dans les deux certificats et à la maîtrise) et un cours d'information juridique (SCI6349 - Sources d'information juridique, cours à option offert à la maîtrise). En outre, nombre de questions éthiques et juridiques sont examinées ponctuellement dans différents cours.

Je m'en tiendrai ici à la présentation des deux cours susmentionnés, afin de présenter mon approche pédagogique du droit dans le milieu particulier des SI.

\section{Aspects juridiques des archives et de l'information (ARV3053)}

Le cours « Aspects juridiques des archives et de l'information " s'adresse à un auditoire hétérogène, dans la mesure où il est offert en option aux étudiants des programmes de $1^{\text {er }}$ et de $2^{\mathrm{e}}$ cycles de l'EBSI depuis l'hiver 2014. Les deux premières années, il a été offert en soirée, avec une certaine proportion d'étudiants déjà actifs dans la vie professionnelle au sien de milieux archivistiques ou de bibliothèques. Chacun vient ainsi avec son propre parcours, des niveaux d'expérience variables et des questionnements juridiques spécifiques et variés. Rares sont les étudiants inscrits à ce cours qui ont un parcours ou une expérience préalable du droit. D’entrée de jeu, la composition de l'auditoire constitue un défi pédagogique stimulant, dans la mesure où il faut être en mesure de captiver l'attention de tout le monde et de ne perdre personne en route.

Partant de ce constat, le cours vise à donner un certain nombre de clés de lecture du droit de l'information, afin que chacun dispose des outils pour comprendre les enjeux juridiques de son (futur) milieu professionnel. Chemin faisant, il convient d'éviter à la fois le piège du jargon gratuit et intimidant, mais aussi celui d'une vulgarisation excessive et simplificatrice. Si l'objectif du cours n'est pas de transformer les étudiants en avocats, il s'agit de leur donner accès à des principes et à des termes juridiques clairs et précis, pour qu'ils les comprennent et puissent les utiliser à bon escient. Le cours s'adresse à des étudiants non-initiés et ne nécessite pas de formation préalable en droit. 
Dans cette perspective, le cours s'articule en deux étapes qui balisent le cheminement de l'étudiant dans l'environnement juridique. Ainsi, il propose d'abord une initiation à l'information juridique elle-même comme outil de compréhension du droit, avant de plonger dans le droit de l'information à proprement parler. Ces deux étapes correspondent aux deux principaux objectifs pédagogiques du cours, à savoir la compréhension de l'information juridique et la compréhension du droit de l'information.

\section{Premier objectif pédagogique : comprendre l'information juridique}

Le fait de consacrer une première partie du cours à l'information juridique semble naturel, surtout dans un programme de formation en SI. Une telle entrée en matière vise à montrer aux étudiants à quel point le droit et les SI convergent, dans la mesure où le droit ne serait rien sans les sources qui constituent son fondement, à savoir la loi, la jurisprudence et la littérature scientifique (appelée doctrine). Ces sources intellectuelles du droit se matérialisent nécessairement dans des ressources informationnelles, qui sont à la fois un objet bien connu du professionnel de l'information et un outil de travail indispensable pour le juriste.

\section{Compétences à développer par l'étudiant}

Le but de cette partie du cours est d'abord de démystifier le droit, qui peut intimider, voire bloquer les étudiants non-initiés. En donnant des clés d'accès aux ressources juridiques, on permet aux étudiants de comprendre l'information juridique et son importance comme source du droit. L'étudiant sera ainsi capable de distinguer les différentes sources du droit (législation, jurisprudence, doctrine), de comprendre l'organisation de l'information juridique et de trouver l'information juridique pertinente. Il développera ainsi de bons réflexes juridiques, qui consistent à consulter l'information juridique par soi-même (et non, par exemple, par l'entremise de la presse) en allant directement aux sources et en étant capable de les déchiffrer raisonnablement. Il pourra aussi mettre à jour les connaissances acquises dans le cadre du cours, sachant que le droit évolue rapidement.

\section{Activités d'apprentissage}

Après un exposé théorique et introductif sur les sources et principes généraux du droit, l'étudiant est rapidement invité à passer à l'action. Dès le second cours, un exercice d'exploration de la bibliothèque de la faculté de droit est organisé pour «briser la glace ». L'exercice se veut ludique autant que didactique : armés d'un questionnaire, du plan de classification ${ }^{4}$ et du plan de la bibliothèque, les étudiants déambulent dans les rayons pour y localiser des ouvrages précis qu'ils devront manipuler et explorer afin de répondre aux questions. Cette activité est généralement très appréciée des étudiants, qui peuvent ainsi « voir » et " toucher » le droit, c'est-à-dire l'inscrire dans un système organisé et une réalité concrète directement en lien avec l'exposé théorique.

La seconde activité pédagogique en lien avec la compréhension de l'information juridique consiste en un exercice de recherche dans les bases de données juridiques. Partant du principe que la majorité des étudiants ne disposeront pas, dans leur environnement professionnel, d'un accès aux bases de données juridiques payantes, l'exercice se focalise sur les bases de données québécoises et canadiennes en libre accès ${ }^{5}$. Le but est de démontrer à l'étudiant qu'à travers ces ressources accessibles à tous, il dispose déjà d'outils assez performants pour repérer l'information juridique de base. Pour aider l'étudiant à s'orienter dans le dédale des bases de données, un petit module accessible sur le site Web du cours leur propose un parcours en fonction de l'information recherchée (législation fédérale ou québécoise, loi ou règlement, projet de loi ou de règlement, jurisprudence et doctrine) et qui les redirige vers les sites Web appropriés par le jeu d'hyperliens, avec des explications de base sur la manière de formuler une requête juridique.

Enfin, un recueil de législation est fourni aux étudiants afin de les amener à consulter régulièrement les textes. Ce recueil contient les textes de loi relatifs aux aspects juridiques des archives et de l'information qui sont exposés au cours. Tout au long de la session, les étudiants sont invités à utiliser leur recueil pour réviser leurs notes, à souligner les passages importants et à en marquer les pages, afin d'y retrouver facilement l'information et ainsi faire le lien entre le cours et les textes. Lors de l'examen, leur capacité à trouver l'information juridique dans le recueil de textes est mise à l'épreuve, à travers une série de questions qui les obligent à trouver et à comprendre les dispositions pertinentes dans les lois.

4. La bibliothèque de droit de l'Université de Montréal présente l'intérêt d'utiliser une classification « maison " qui tient compte des trois types de sources, des différentes branches du droit et de la juridiction concernée. Cette subdivision est particulièrement intéressante d'un point de vue pédagogique, en permettant aux étudiants de revoir les fondements du droit présentés dans le cours à travers l'outil familier du plan de classification.

5. Notamment CanLII (<www.canlii.org $>$ ), CAIJ ( $<$ www.caij.qc.ca $>)$, Publications du Québec (législation québécoise) ( $<$ www3.publicationsduquebec.gouv.qc.ca/ loisreglements.fr.html>), Justice Canada (législation fédérale) (<laws-lois.justice. gc.ca $>$ ), mais aussi les travaux de l'Assemblée nationale ( $<$ www.assnat.qc.ca $>$ ), la base de données LEGISInfo du Parlement canadien (<www.parl.gc.ca/LegisInfo $>$ ), etc. 
Deuxième objectif pédagogique : comprendre le droit de l'information

Après cette mise en condition, les étudiants sont prêts à entrer dans le vif du sujet et à explorer le droit de l'information et des archives. Il convient de donner aux professionnels les outils pour pouvoir appliquer les règles en matière de protection des informations à caractère personnel, de propriété intellectuelle (droit d'auteur et droits voisins, protection des bases de données, licences et logiciels libres) et de droit d'accès aux informations du secteur public, sans oublier la Loi sur les archives. Ces dispositions sont en effet directement utiles dans leur pratique quotidienne, pour gérer la sécurité, la confidentialité, la reproduction, la diffusion et l'accessibilité des documents, de même que leur destruction, que ce soit en interne ou vis-à-vis du public ou d'un co-contractant. Rappelons que ces règles ont toutes fait l'objet d'adaptations pour tenir compte de l'environnement numérique, où elles suscitent de profonds questionnements. En outre, pour pouvoir encadrer le contexte de création et de conservation des documents tout au long de leur cycle de vie de manière appropriée, il est nécessaire d'avoir des éléments de droit de la preuve, y compris à l'heure du numérique (principes généraux et conditions de la reconnaissance juridique des documents et des signatures électroniques), et d'être sensibilisé à l'existence de règles générales et spécifiques pour la validité et la conservation des documents. Dans le contexte plus global de la formation archivistique, on peut encore y ajouter des notions de droit des contrats pour pouvoir comprendre et respecter les conventions qui entourent le versement et la cession de fonds d'archives, ainsi que le dépôt légal. Enfin, les questions d'éthique et de déontologie professionnelle sont naturellement de la plus grande importance. Telles sont les matières juridiques abordées dans le cadre du cours. Étant donné que le cours s'inscrit dans l'offre de cours du certificat en archivistique (ARV), on constate qu'une partie importante est consacrée au droit des archives.

\section{Compétences à développer par l'étudiant}

L'objectif de cette partie du cours est que l'étudiant soit capable de comprendre l'impact du droit sur l'information en général, et sur les archives et les fonctions archivistiques en particulier. Plus spécifiquement, l'étudiant doit pouvoir :

- comprendre les grands principes du droit d'auteur et leur application aux informations et aux archives;

- comprendre les grands principes du droit de la preuve et de la loi sur les technologies de l'information et leurs impacts sur la gestion de l'information et des archives;
- comprendre la législation sur la protection des renseignements personnels et sur l'accès aux documents des organismes publics;

- gérer l'accès à l'information et aux archives dans le respect de la législation;

- comprendre un contrat de donation d'archives;

- comprendre ce qu'est le dépôt légal;

- identifier les exigences juridiques pour la conservation de l'information et des archives, ainsi que les enjeux de la préservation numérique;

- appliquer la législation sur les archives;

- mesurer la dimension éthique de la pratique archivistique et respecter la déontologie professionnelle de l'archiviste.

En outre, l'étudiant doit être capable de saisir les enjeux sociétaux du droit et de développer un regard critique sur le droit et ses limites.

\section{Activités d'apprentissage}

Cette partie du cours s'appuie sur une pédagogie plus classique, faite d'exposés ex cathedra de la professeure et de conférences données par des experts extérieurs. En outre, afin d'illustrer les principes vus au cours, un travail individuel sur une actualité juridique est mis en place. Il consiste à demander aux étudiants de choisir un article (blogue, article de presse, etc.) qui traite d'une actualité juridique en lien avec les matières vues dans le cours (une nouvelle législation, un procès médiatisé, etc.). Les étudiants doivent être capables de contextualiser l'événement, de trouver la législation ou la jurisprudence concernée et de poser un regard critique sur l'actualité. L'exercice les invite à faire le lien avec les principes vus dans le cours et à aller au-delà de l'article de presse pour trouver et comprendre l'information juridique. Il leur permet aussi de découvrir certains sites Web ou blogues spécialisés.

Un autre travail pratique concerne plus spécifiquement les obligations légales d'archivage. Dans le cadre de leur pratique et à travers le calendrier de conservation, les archivistes appliquent diverses obligations légales de conservation de documents. L'exercice consiste à choisir deux types de documents dont la conservation est obligatoire en vertu d'une obligation légale ou réglementaire, dans deux secteurs d'activités différents. Pour chaque document, les étudiants doivent trouver et analyser la disposition légale qui impose sa conservation : son champ d'application, la raison pour laquelle la conservation est imposée, les contraintes juridiques en termes de délais, de support, de modalités de conservation, d'accès, ainsi que les éventuels conflits avec d'autres règles de conservation. Ils sont ainsi amenés à s'interroger sur la règle de conservation, ses fondements et sa raison d'être, tout en développant un regard critique sur le texte de loi, souvent insuffisant ou incomplet quant aux modalités de création et aux conditions de conservation des documents; ces moda- 
lités doivent souvent être explicitées, voire complétées, par les archivistes eux-mêmes.

\section{Sources d'information juridique (SCI6349)}

L'informatique juridique est un champ de compétence qui gagne à être développé en SI. Elle consiste en l'étude de l'information juridique, des technologies qui la sous-tendent et de son impact en droit (Hinson 2005, 134). Le cours "Sources d'information juridique », offert en option à la maîtrise, vise à permettre aux étudiants de trouver et de comprendre l'information juridique, mais aussi de développer une bonne connaissance des défis et des enjeux de la bibliothéconomie juridique, à travers la rencontre des milieux professionnels. Il ne s'adresse pas seulement aux étudiants qui veulent s'orienter vers les bibliothèques spécialisées, mais également à ceux qui souhaitent développer une compétence de recherche dans les bases de données juridiques spécialisées et la valoriser sur le marché du travail.

Le cours n'exige pas de prérequis en droit et s'adresse à des non-juristes. Il est par contre recommandé d'avoir réussi le cours obligatoire "Sources et recherche d'information »(SCI6057), qui fait partie du tronc commun dans le programme de MSI.

\section{Premier objectif pédagogique : trouver et comprendre l'information juridique}

Dans le cours "Aspects juridiques des archives et de l'information ", l'enseignement des bases de l'information juridique visait à permettre aux étudiants d'accéder au droit de l'information en passant par le chemin familier de l'information juridique elle-même. Dans le cours "Sources d'information juridique ", la démarche est différente, puisque l'information juridique n'est pas un outil pédagogique, mais l'objet même du cours.

\section{Compétences à développer par l'étudiant}

Avant de pouvoir trouver l'information juridique, l'étudiant doit pouvoir comprendre, d'une part, l'organisation d'un système juridique et, d'autre part, l'organisation de l'information juridique elle-même.

Au terme du cours, l'étudiant sera capable de comprendre les sources du droit (législation, jurisprudence, doctrine) et les systèmes juridiques canadien et québécois. Il pourra également distinguer les différentes familles juridiques (common law vs droit civil) et se repérer dans d'autres systèmes juridiques, comme celui des États-Unis ou de l'Union européenne. Il pourra s'orienter dans l'architecture de l'information juridique, en étant capable d'utiliser les principales bases de données juridiques québécoises, canadiennes et étrangères en libre accès ou payantes. Enfin, il sera capable de lire, comprendre et écrire une référence juri- dique, de trouver l'information juridique, de répondre à une demande d'information juridique et d'organiser, contextualiser et analyser l'information juridique trouvée.

\section{Activités d'apprentissage}

Étant donné qu'il s'agit d'un auditoire de nonjuristes, une initiation et une sensibilisation en douceur à l'univers juridique est nécessaire en guise d'entrée en matière. On ne s'étonnera pas de revoir, au début du cours, des activités pédagogiques similaires à celles qu'on trouve également dans le cours «Aspects juridiques des archives et de l'information ", mais dans une forme plus approfondie : des exposés théoriques relatifs aux systèmes juridiques québécois et canadien, une exploration de la bibliothèque de droit et de son catalogue ${ }^{6}$, des formations spécifiques aux principales bases de données juridiques québécoises payantes ${ }^{7}$ et en libre accès $^{8}$.

Outre les formations à l'utilisation des bases de données, des laboratoires et des exercices en bibliothèque sont organisés afin de permettre à l'étudiant de faire une recherche poussée en législation (y compris dans les travaux préparatoires des lois), en jurisprudence et en doctrine. Les exercices sont conçus pour un apprentissage individuel, autonome et actif de l'étudiant, qui est invité à explorer par lui-même l'environnement de recherche à travers un questionnaire, tout en étant guidé par les informations et les exemples nécessaires pour comprendre le contexte juridique et les procédures à suivre.

Au terme du cours, les étudiants sont amenés à synthétiser leurs compétences par deux travaux individuels complémentaires. Le premier repose sur le modèle de la "liste d'épicerie ": dans la pratique professionnelle, il est fréquent que l'on demande aux bibliothécaires juridiques de trouver des documents à partir d'une liste bibliographique plus ou moins précise. À partir des références de doctrine et de jurisprudence qui leur sont fournies de manière incomplète, les étudiants doivent faire une recherche dans les cinq bases de données présentées durant le cours, pour comparer les résultats obtenus et vérifier s'ils peuvent retrouver le document et, le cas échéant, accéder à une version intégrale du texte. Le second travail consiste à effectuer une recherche pour constituer une bibliographie juridique sur un sujet imposé. L'étudiant doit développer et expliquer sa stratégie de recherche pour trouver des documents de référence dans les bases de données juridiques, évaluer ses résultats de recherche et expliquer les

6. Catalogue de l'Université de Montréal, Atrium (<atrium.umontreal.ca>).

7. SOQUIJ (Azimut), Lexis Nexis Quicklaw, Westlaw Canada et La Référence.

8. CanLII (<www.canlii.org >), CAIJ (<www.caij.qc.ca $>$ ), Publications du Québec (législation québécoise) ( $<$ www3.publicationsduquebec.gouv.qc.ca/loisreglements.fr.html $>$ ), Justice Canada (législation fédérale) (<laws-lois.justice.gc.ca $>$ ), les travaux de l'Assemblée nationale ( $<w w w . a s s n a t . q c . c a>)$ et la base de données LEGISInfo du Parlement canadien (<www.parl.gc.ca/LegisInfo $>$ ). 
raisons de ses choix bibliographiques, et enfin organiser l'information juridique de manière cohérente et la citer de manière conforme aux règles de citation juridiques.

\section{Deuxième objectif pédagogique : comprendre le contexte professionnel de l'information juridique}

\section{Compétences à développer par l'étudiant}

Il serait quelque peu austère de limiter le cours aux aspects techniques de la recherche d'information juridique. C'est pourquoi le second objectif du cours est d'ancrer l'information juridique dans la réalité professionnelle et de l'incarner dans des hommes et des femmes qui peuvent témoigner de parcours professionnels et d'environnements juridiques éclairants pour l'étudiant. En lien avec ces objectifs, les étudiants sont amenés à comprendre les métiers du droit et les différents parcours des professionnels de l'information dans le milieu juridique, et à développer un regard critique sur le milieu professionnel et ses possibles débouchés.

\section{Activités d'apprentissage}

Trois visites en milieu professionnel ont pu être organisées à l'hiver 2015 dans le cadre du cours. Les visites présentent, sur les simples conférences en classe, l'avantage d'une immersion sur le terrain et de rencontrer plusieurs responsables et employés de la même institution. Ainsi, il nous a été possible de visiter le centre de documentation et de ressources informationnelles de la Chambre des notaires, le service d'information et la bibliothèque d'un grand cabinet d'avocats et le Centre d'accès à l'information juridique (CAIJ). Les trois milieux ont offert un excellent accueil et des explications nourries sur leur travail. Ces visites ont obtenu un franc succès auprès des étudiants, qui les ont trouvées riches en enseignements.

À la suite de chaque visite, un rapport de visite ${ }^{9}$ d'une à deux pages doit être rédigé par l'étudiant. Ce rapport présente brièvement le milieu professionnel visité et fait part des impressions personnelles de l'étudiant. Il s'agit pour l'étudiant de démontrer sa compréhension du lieu et du travail de gestion de la documentation juridique qui y est effectué, et de faire montre d'esprit critique et de curiosité professionnelle.

\section{Conclusion}

Le milieu professionnel et les étudiants en SI expriment un intérêt grandissant pour le droit. Le droit de l'information, en particulier, est en pleine expansion avec les questionnements nouveaux et complexes suscités par l'environnement numérique qui ne manquent pas de surgir dans la pratique professionnelle. Par ailleurs, le volume d'information juridique a atteint des proportions telles que la gestion même de cette information, son exploitation et sa mise à jour suscitent, tant auprès des grands éditeurs juridiques qu'auprès des défenseurs du savoir libre, de nouveaux défis que les juristes ne pourront pas résoudre sans l'aide des professionnels de l'information. Il y a là assurément une niche de compétences à développer.

L'intégration du droit dans les programmes d'études en SI semble progresser. Cependant, le développement de nouveaux profils et de nouvelles spécialisations nécessite d'aller au-delà d'un ou de deux cours à option dans le parcours de l'étudiant. L'offre de diplômes conjoints en droit et en SI est une voie à explorer. Déjà bien ancrée aux États-Unis, l'idée doit encore faire son chemin au Canada, et en particulier au Québec. Il convient toutefois de veiller à l'établissement de programmes suffisamment intégrés et adaptés, afin d'éviter la simple juxtaposition de cours, sans tenir compte des réelles interactions entre les deux disciplines et sans adapter la pédagogie du droit aux non-juristes (et, inversement, la pédagogie des SI aux juristes).

Il est possible, au moyen d'une pédagogie adaptée, de mettre le droit à la portée des non-initiés et de le rendre attractif. Une fois familiarisés avec l'environnement juridique, ses sources, ses méthodes, ses acteurs et sa terminologie, les étudiants y évoluent avec une certaine aisance et souvent avec intérêt, voire étonnement. En questionnant le droit à travers leur pratique et leur vision disciplinaire, ils participent à son enrichissement et à sa mise en pratique. En développant des repères, des réflexes et des connaissances juridiques, ils acquièrent de nouvelles compétences qui leur seront précieuses, non seulement sur le marché du travail, mais aussi en tant qu'individu dans la société. Le droit ne peut plus se contenter d'être une discipline de caste. C'est un savoir qui doit être mis à la portée du plus grand nombre, afin d'en assurer une meilleure application, de faciliter le dialogue interdisciplinaire et de comprendre les enjeux sociétaux du droit. -

9. L'outil pédagogique du rapport de visite s'inspire du " rapport d'étonnement " utilisé par certains collègues de l'EBSI (Maurel et al. 2006, 60). 


\section{Sources consultées}

Couture, Carol \& Marcel Lajeunesse. 2014. L'archivistique à l'ère du numérique: les éléments fondamentaux de la discipline. Québec: Presses de l'Université du Québec.

Demoulin, Marie \& Sébastien Soyez. 2015. L'interdisciplinarité dans la formation archivistique : un atout pour l'archiviste de demain. In Archivistes de 2030 - Réflexions prospectives, sous la direction de Paul Servais, avec la collaboration de Françoise Mirguet. Louvain-la-Neuve : Academia (Publications des archives de l'Université catholique de Louvain, 32), 157-169.

Demoulin, Marie \& Sébastien Soyez. 2014. Law and records management in archival studies: New skills for digital preservation. In Making the Information Governance Landscape in Europe, Proceedings of the $7^{\text {th }}$ Triennial Conference of the DLM Forum, sous la direction de José Borbinha, Zoltàn Szatucsek \& Seamus Ross. Lisbonne : Biblioteca Nacional de Portugal, 131-135. $<$ purl.pt/26107/1/DLM2014_PDF/dlm2014-Proceedings_ V1.pdf $>$ (consulté le 10 mai 2015).

Fernández-Molina, Carlos. 2004. Legal aspects of electronic information in the training of information professionals. Journal of Education for Library and Information Science 45 (2) : 111-122.

Gathegi, John N. \& Darrell E. Burke. 2008. Convergence of information and law: A comparative study between i-schools and other ALISE schools. Journal of Education for Library and Information Science 49 (1) : 1-22.

Maurel, Dominique, Zeïneb Gharbi, Sabine Mas, Luce Payette, Lyne Da Sylva \& Éric Leroux. 2006. Les pratiques pédagogiques exemplaires en sciences de l'information : 21 fiches annotées. Comité local d'intégration pédagogique de l'EBSI. Université de Montréal. <clip.ebsi.umontreal.ca/documents/pratiques_ v2_2006.pdf> (consulté le 15 mai 2015).

Prégent, Richard. 2009. Enseigner à l'université dans une approcheprogramme : guide à l'intention des nouveaux professeurs et chargés de cours. Montréal : Presses internationales Polytechnique. 